\title{
ÉTUDE DE LA VALEUR ALIMENTAIRE DU MAIS-GRAIN SÉCHÉ ARTIFICIELLEMENT POUR LA CROISSANCE DU POUSSIN. II. - INFLUENCE DE LA DURÉE QUI SÉPARE LA RÉCOLTE DU SÉCHAGE
}

\author{
PAR \\ C. CALET et H. TARDIF. \\ Station de Recherches Avicoles, C. N. R. Z., Jouy-en-Josas (S.-et-O.).
}

SOMMAIRE

La durée qui sépare la récolte du séchage du maïs égréné se traduit par une fermentation qui aboutit à une diminution de $20 \mathrm{p}$. Ioo de l'efficacité du grain. Les modifications chimiques du maïs, responsables de cette altération, n'apparaissent pas à l'analyse. Toutefois l'addition de $0,08 \mathrm{p}$. Ioo de tryptophane au régime remédie complètement à l'abaissement de la valeur alimentaire du grain sous l'effet de la fermentation.

Une première étude (CALET et de LAMBILLY, Ig60) a permis de préciser l'influence de la température de séchage du maïs sur sa valeur alimentaire et sur la disponibilité de son tryptophane ou de sa niacine. Lorsque la température de séchage s'élève jusqu'à $90^{\circ} \mathrm{C}$, aucune modification de la valeur alimentaire dı grain n'est enregistrée. Par contre, des grains qui ont fermenté avant le séchage présentent une efficacité alimentaire nettement abaissée, bien que la disponibilité du système tryptophane-niacine paraisse accrue.

Les méthodes modernes de récolte du maïs consistent de plus en plus à égrener l'épi. Elles obligent à sécher artificiellement le grain en vue de sa conservation. Toutefois, il s'écoule un temps plus ou moins variable entre les moments où les grains sont prélevés sur la plante, et où ils sont suffisamment secs. Pendant ces durées, le grain est le siège de fermentations importantes, puisque, au sein des tas, la température peut s'élever jusqu'à $80^{\circ}$. 
Afin de déterminer l'importance et la nature des pertes apparues au cours de la fermentation, nous avons étudié les modifications de la valeur alimentaire des grains soumis à des durées contrôlées de fermentation.

\section{MA'TÉRIEL ET MÉTHODES}

Nous disposons de trois échantillons de maïs de la variété INRA 200 cultivés, sur le même sol, et récoltés le même jour. Le lot $\mathrm{n}^{0}$ I est séché immédiatement; le second, au bout de 24 heures de stockage en tas, et le troisième au bout de $4^{8}$ heures. La température de séchage est fixée à $45^{\circ} \mathrm{C}$ afin de limiter la durée de $1^{\prime}$ 'opération et ainsi de ne pas modifier l'évolution des fermentations, sans provoquer toutefois d'altération sous l'effet d'une température trop élevée. Les grains possèdent à la fin du traitement une humidité de I4 p. Ioo environ. Les durées de séchage sont de l'ordre de I8 heures. La composition fourragère et l'analyse des différents acides aminés du maïs ne révèlent pas de différences notoires entre les échantillons, comme le montre le tableau I.

TABLEAU I

Composition des échantillons de maïs (1).

\begin{tabular}{|c|c|c|c|}
\hline $\begin{array}{c}\text { Maïs } \\
\text { Durée de fermentation }\end{array}$ & $o^{I} h$ & $\begin{array}{c}\text { II } \\
24 \mathrm{~h}\end{array}$ & ${ }_{48 \mathrm{~h}}^{\text {III }}$ \\
\hline 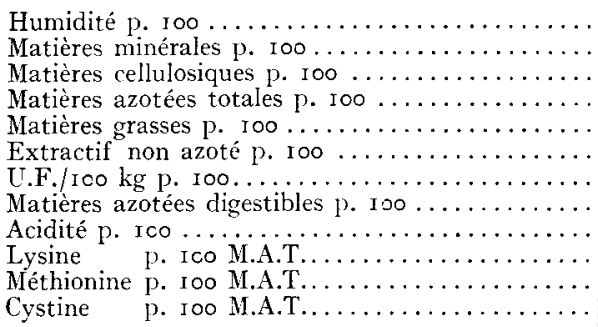 & $\begin{array}{l}\mathrm{I}, 3,9 \\
\mathrm{I}, 43 \\
2,2 \\
9,5 \\
5, \mathrm{I} \\
67,9 \\
\mathrm{I} \mathrm{I} 4 \\
6,8 \\
0,2 \\
2,5 \mathrm{I} \\
\mathrm{I}, 95 \\
\mathrm{I}, \mathrm{I}\end{array}$ & $\begin{array}{l}\mathrm{I} 3,7 \\
\mathrm{I}, 43 \\
2, \mathrm{I} \\
8,9 \\
5,2 \\
68,7 \\
\mathrm{I} \mathrm{I} \\
6,4 \\
0,25 \\
2,57 \\
\mathrm{I}, 88 \\
\mathrm{I}, \mathrm{I} 3\end{array}$ & $\begin{array}{r}\mathrm{r} 3,6 \\
\mathrm{I}, 44 \\
\mathrm{I}, 87 \\
9, \mathrm{I} \\
\mathbf{5}, 6 \\
68,3 \\
\mathrm{I} \mathrm{I} 5 \\
6,5 \\
0,2 \mathrm{I} \\
2,72 \\
\mathrm{I}, 94 \\
\mathrm{I}, 05\end{array}$ \\
\hline
\end{tabular}

On est surpris de ne voir apparaitre à l'analyse aucune différence dans la composition chimique des grains. La fermentation ne s'accompagne ni d'une diminution de la matière sèche, ni de pertes énergétiques, ni d'une élévation corrélative du taux de cellulose. Les teneurs en matières azotées totales et digestibles et en acides aminés dosés ne sont pas modifiées par le traitement.

Les différents échantillons sont incorporés dans les régimes expé-

(1) Les analyses des divers échantillons de maīs ont été effectuées par le laboratoire de Biochimie et de Nutrition du C N R Z. 
rimentaux, au taux de 80 p. Ioo. Ils représentent ainsi la plus grande partie des éléments énergétiques du régime. Afin de mieux mettre en évidence une éventuelle variation dans la disponibilité du tryptophane ou de la nicotinamide au cours de la fermentation, le reste de la ration est constitué des sources azotées très déficientes en tryptophane et la vitamine PP ne figure pas dans le complément vitaminique. Néanmoins, dans la moitié des lots, les régimes sont surchargés d'une dose unique de tryptophane qui couvre largement les besoins de l'animal en cet acide aminé.

I80 poussins mâles, issus du croisement Rhode $\times$ Wyandotte, sont répartis à l'âge de $I 5$ jours en $I 8$ lots homogènes et sont élevés pendant 7 semaines en batteries. I,e schéma expérimental est donné dans le tableau II.

TABLEAU II

Répartition des lots et des régimes.

\begin{tabular}{|c|c|c|}
\hline Lots & Régimes & $\begin{array}{c}\text { Nombre de } \\
\text { répétitions } \\
\text { par lot }\end{array}$ \\
\hline $\mathrm{I} \cdot \cdot$ & R. B. + Mais I $(80 \%)$ séché immédiatement & 3 \\
\hline$\ldots$. & R. B. + Maís II $(80 \%)$ fermenté 24 h. & 3 \\
\hline$\ldots \ldots$ & R. B. + Maïs III $(80 \%)$ fermenté 48 h. & 3 \\
\hline IV $\ldots \ldots \ldots$ & R. B. - Maîs I $(80 \%)+$ Tryptophane $0,08 \%$ & 3 \\
\hline & R. B. + Mais II $(80 \%)+$ Tryptophane $0,08 \%$ & 3 \\
\hline VI.......... & R. B. + Mais III $(80 \%)+$ Tryptophane $0,08 \%$ & 3 \\
\hline
\end{tabular}

La composition du régime de base, qui représente $20 \mathrm{p}$. Ioo des rations expérimentales, renferme les constituants suivants (exprimée en pourcentage) :

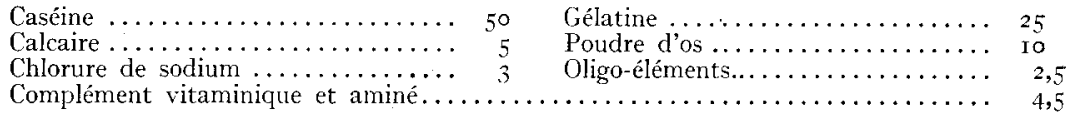

Le complément vitaminique et aminé fournit, pour Ioo kg de régime expérimental, roo $g$ de $\mathrm{DI}_{4}$ méthionine et toutes les vitamines nécessaires au poussin en doses optimales, sauf la nicotinamide qui n'est pas présente. Ainsi les régimes qui titrent I 9 p. Ioo de matières azotées couvrent tous les besoins nutritifs du poussin sauf ceux des facteurs étudiés.

\section{RÉ,SULTATS}

Les résultats portant sur la croissance et la consommation de nourriture sont rassemblés dans le tableau III. 


\section{TABLEAU III}

Influence du temps de fermentation du mais sur la croissance du poussin et sur l'efficacité du régime.

\begin{tabular}{|c|c|c|c|c|c|c|c|}
\hline & \multirow{2}{*}{ Lots } & \multicolumn{3}{|c|}{$\mathrm{A}_{4}$ semaines } & \multicolumn{3}{|c|}{ A 7 semaines } \\
\hline & & $\begin{array}{r}\text { Gain de } \\
\text { poids }(\mathrm{g})\end{array}$ & $\left|\begin{array}{c}\text { Nourriture } \\
\text { ingérée } \\
(\mathrm{g})\end{array}\right|$ & I.C. & $\begin{array}{l}\text { Gain de } \\
\text { poids (g) }\end{array}$ & $\left|\begin{array}{c}\text { Nourriture } \\
\text { ingérée }(\mathrm{g})\end{array}\right|$ & I.C. \\
\hline $\begin{array}{l}\text { Mais I (o heure) .... } \\
\text { Maïs II ( } 24 \text { heures).. } \\
\text { Maïs III ( } 48 \text { heures). }\end{array}$ & $\begin{array}{l}\text { I } \\
\text { II } \\
\text { III }\end{array}$ & $\begin{array}{l}296 \\
279 \\
\mathbf{2 4 8}\end{array}$ & $\begin{array}{l}829 \\
790 \\
831\end{array}$ & $\begin{array}{l}2,80 \\
2,84 \\
3,35\end{array}$ & $\begin{array}{l}590 \\
540 \\
477\end{array}$ & $\begin{array}{l}\text { I } 917 \\
\text { I } 695 \\
\text { I } 755\end{array}$ & $\begin{array}{l}3,25 \\
3,14 \\
3,68\end{array}$ \\
\hline $\begin{array}{l}\text { Maïs I + Tryptophane } \\
\text { Mais II + Tryptophane } \\
\text { Mlaïs III + Tryptophane }\end{array}$ & $\begin{array}{l}\text { IV } \\
\mathrm{V} \\
\mathrm{VI}\end{array}$ & $\begin{array}{l}435 \\
407 \\
410\end{array}$ & $\begin{array}{r}966 \\
964 \\
1012\end{array}$ & $\begin{array}{l}2,22 \\
2,37 \\
2,47\end{array}$ & $\begin{array}{l}937 \\
896 \\
895\end{array}$ & $\begin{array}{l}2605 \\
2527 \\
2586\end{array}$ & $\begin{array}{l}2,78 \\
2,82 \\
2,89\end{array}$ \\
\hline
\end{tabular}

Lorsque le régime n'est pas supplémenté en tryptophane, le mode de préparation du grain retentit considérablement sur sa valeur alimentaire. Plus la durée du stockage du maïs humide est importante, moins la croissance de l'animal est rapide. Les différences observées entre les valeurs extrêmes tant à 4 semaines qu'à 7 semaines sont de l'ordre de $20 \mathrm{p}$. I00. On ne remarque pas de variations importantes dans la consommation du maïs à la quatrième semaine. Par contre, au fur et à mesure que 1'animal vieillit, l'appétance des différents mais est modifiée et la fermentation conduit à une diminution de la quantité d'aliment ingéré. Il en résulte, dans tous les cas, une élévation de l'indice de consommation, qui est toutefois plus marquée lorsque l'animal est jeune. Il faut souligner que ce sont les maïs les plus fermentés qui fournissent les indices de consommation les moins recherchés.

L'effet le plus spectaculaire est consécutif à l'addition de tryptophane. On assiste en effet à une augmentation considérable de la vitesse de croissance qui est presque doublée aussi bien à 4 qu'à 7 semaines. $L_{1} a$ supplémentation des régimes distincts par une dose élevée de tryptophane remédie entièrement aux déficiences des divers échantillons de maïs quelle que soit leur origine. Les différences très faibles que l'on enregistre dans les gains de poids ne sont pas significatives même à 7 semaines. De son côté, l'indice de consommation est également amélioré, mais il reflète encore la hiérarchie des valeurs alimentaires des maīs, primitivement établie.

\section{DISCUSSION}

On pouvait s'attendre à ce que, au cours du stockage du maïs humide, la fermentation se traduise par une perte des constituants de la céréale, et plus spécialement des principes énergétiques. En fait, l'analyse 
chimique montre qu'il n'en est rien, puisque la valeur fourragère des divers échantillons est rigoureusement la même. Cette observation est à rapprocher de celle d'ARchibald et al., I95I, qui montre que la valeur énergétique d'un fourrage ne s'abaisse qu'au bout de plusieurs semaines de fermentation. Ce phénomène n'apparait que dans la mesure oì la teneur en matière sèche de la plante est diminuée. Pourtant les réponses de croissance enregistrées révèlent les différentes aptitudes des maīs. Il semble que le mais subisse une diminution de sa valeur alimentaire au cours de la fermentation qui porte sur une perte de tryptophane. En effet l'adjonction d'une large quantité de tryptophane fait disparaître les inégalités d'efficacité des maïs et remédie également à la déficience des divers régimes. On connaît le rôle du tryptophane dans la synthèse de la nicotinamide. ROSEN etal., I946, montrent que l'adjonction de tryptophaneau régime provoque une élimination accrue des dérivés de la vitamine PP. De plus, le besoin en tryptophane s'accroît lorsque la ration est déficiente en vitamine PP. Le tryptophane serait donc un précurseur de cette vitamine qui est souvent indisponible dans les aliments. C'est le cas en particulier du maïs.

KodiceK, BraUde, Kon et Mitcheli, I959, ont dosé les vitamines B dans le maiss traité selon différents modes de préparation. La teneur en vitamine $P P$ libre du maïs non traité est très faible $(I, 7 \mathrm{p}$. Ioo de la vitamine totale). Elle devient par contre très élevée lorsque le maïs a été cuit dans l'eau de chaux ( $98 \mathrm{p}$. roo de la vitamine totale). La modification de la valeur alimentaire du grain réside dans la mise en disponibilité de la nicotinamide et non pas du tryptophane dont la teneur demeure constante $(0,85 \mathrm{p}$. Ioo dans les deux cas). La libération de la vitamine PP des complexes où elle se trouve liée dépend essentiellement de la manière dont le maïs est préparé. Ainsi KODICEK et WILSON, I959, étudiant la valeur alimentaire de "tortilla " obtenus par deux recettes différentes après trempage du maïs dans l'eau de chaux, observent dans l'un des cas, une hydrolyse partielle et une libération incomplète de la vitamine PP. D'un autre point de vue, LAGUNA et CARPENTER, I952, montrent que le simple trempage dans l'eau n'est pas suffisant pour valoriser le maïs. Pour cela une hydrolyse doit être effectuée qui doit porter sur les fractions non glucidiques du grain. Ce sont vraisemblablement ces deux dernières raisons qu'il faut invoquer pour expliquer le fait que la fermentation des grains de maïs n'a pas conduit à une élévation de leur valeur alimentaire, selon notre hypothèse de départ.

\section{SUMMARY}

Field-shelled corn during the course of harvesting is an ever more spreading technique, although it makes it necessary to have corn dried artificially.

In an earlier study, it has been shown that the temperature of drying did not affect the nutritive value of corn if it did not tise above $90^{\circ} \mathrm{C}$. 
The present work reports the effects on corn efficiency of the length of time between harvesting and drying. The longer the period, the longer corn ferments. This fermentation is shown by a decrease in efficiency.

The addition of $0,08 \mathrm{p}$. Ioo of tryptophane overcomes completely the unfortunate effect of fermentation.

\section{RÉFÉRENCES BIBLIOGRAPHIQUES}

Archibald J. G., Bar'T J., Blaisdeli M. L. et Spelman A. F., I95I. Quality in roughages. I.-Factors which influence hay composition and quality. J. Dairy Sci., 34, 656-668.

Calet C. et de Lambiliy H., I960. Étude de la valeur alimentaire du maïsgrain séché artificiellement pour le poussin en croissance. I.-Influence du mode de séchage sur la disponibilité des acides aminés. Ann. Zoot., 9, I8I-I84.

Kodicek E., Braude R., Kon S. K. et Mitcheil, K. G., I959. The availability to pigs of nicotinic acid in tortilla baked from maize traited with lime-water. Brit. J. Nutr., 13, 363-384.

KODICEK E. et WILSON P. W., I959. The availability of bound nicotinic acid to the rat. I.-The effect of lime-water treatment of maize and subsequent baking into tortilla. Brit. J. Nutr., 13, 4I8-430.

LAGUna J. et Carpenter K. J., I95I. Raw versus processed corn in niacindeficient diets. J. Nutr., 45, 2I-28.

Rosen F., HUfF J. W. et PerizWeig W. A., I946. 'The effect of tryptophane on the synthesis of nicotinic acid in the rat. J. Biol. Chem., 163, 343344 . 